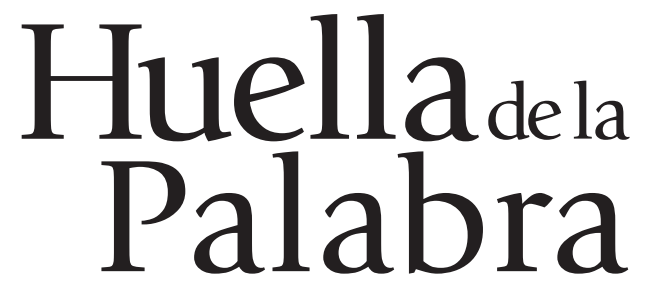

Revista de Investigación Educativa

Universidad La Salle Pachuca

lahuelladelapalabra@lasallep.edu.mx

Teléfono: 01(771) 7170213 ext. 1406

Fax: 01(771) 7170309

ISSN: En trámite

México

Gómez Alvarado Jessica

Vázquez Monter Silvia Carolina

\title{
Coeficiente intelectual en niños de edad escolar con antecedentes de prematurez y asfixia perinatal
}

Huella de la Palabra, año 2015, número 9

Universidad La Salle Pachuca

pp. $71-82$ 



\section{Coeficiente intelectual en niños de edad escolar con antecedentes de prematurez y asfixia perinatal}

Gómez Alvarado Jessica

Vázquez Monter Silvia Carolina

Resumen

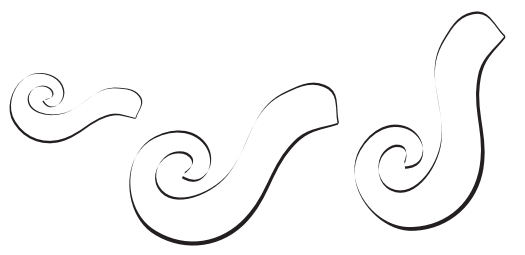

La presente investigación tuvo como objetivo identificar la posible existencia de alteraciones referentes al coeficiente intelectual en niños de edad escolar con antecedentes de prematurez y presencia de asfixia perinatal. La muestra estuvo conformada por 18 niños de edad escolar pacientes del área de Psicología del Centro de Rehabilitación Integral de Hidalgo. Los instrumentos de evaluación utilizados fueron las escalas de inteligencia WPPSI - III (2011), y WISC - IV (2007). El tipo de estudio fue mixto, por lo que fue realizado tanto un análisis cuantitativo como un cualitativo. Para el primero de estos análisis fue usada la estadística descriptiva así como inferencial, mediante la prueba no paramétrica de Kruskal-Wallis, igualmente se utilizó el estadígrafo de contraste $\chi^{2}$. Respecto al análisis cualitativo se empleó la técnica de análisis documental. A través del análisis de los resultados fue posible aceptar la hipótesis de investigación, confirmando así la existencia de alteraciones en el coeficiente intelectual específicamente en el índice de velocidad de procesamiento de los niños de edad escolar con antecedentes de prematurez y presencia de asfixia perinatal.

Palabras clave: coeficiente intelectual, asfixia perinatal, prematurez, escalas weschler

\section{Abstract}

The present study aimed to identify the possible existence of changes concerning the IQ of school- age children with a history of prema-

turity and perinatal asphyxia presence. The sample consisted of 18 school-age children Psychology area patients of the Integral Rehabilitation Center of Hidalgo. The assessment instruments were used intelligence scales WPPSI - III (2011), and WISC - IV (2007). The type of study was mixed, so that was conducted both a quantitative as qualitative. For the first of these analyzes was used descriptive statistics and inferential, using nonparametric Kruskal - Wallis, also used the contrast statistic $\chi^{2}$. The qualitative analysis technique was used documentary analysis. Through the analysis of the results was possible to accept the research hypothesis, thus confirming the existence of alterations in IQ specifically on the processing speed index of school-age children with a history of prematurity and perinatal asphyxia presence.

Keywords: intelligence quotient, perinatal asphyxia, prematurity, weschler scales

\section{Introducción}

De acuerdo a datos otorgados por la Organización Mundial de la Salud, actualmente las infecciones, la prematuridad y la asfixia perinatal representan las tres principales causas de mortalidad neonatal al tener un $23 \%$ dentro de las causas globales (Delfino, Weinberg, Delucchi, Del Campo, Bargueño, Filgueira, Giró, Grajales, Juncal, Kessler, Lanwagen, Mancuello, Marichal, Vargas, Yanes, Bengoa, Berta y Scavone, 2010). 


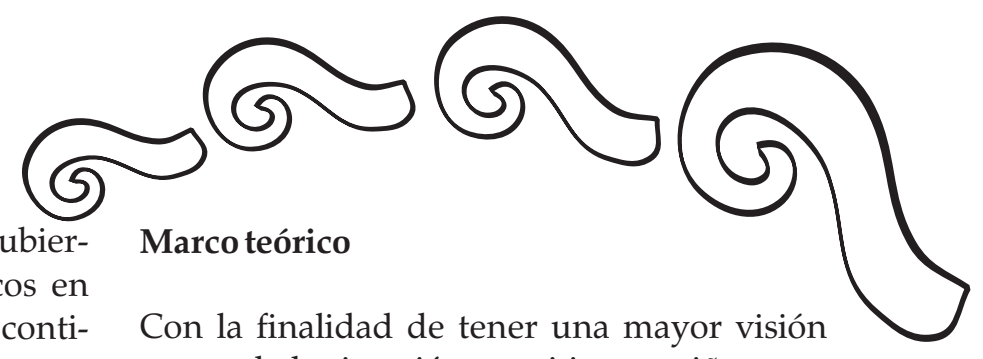

Al tomar en cuenta lo anterior, se ha descubierto que aún con los avances tecnológicos en cuanto a monitoreo fetal, actualmente continúa en aumento la incidencia de casos de patologías fetales y neonatales, especialmente en cuanto a la asfixia perinatal. Cabe mencionar que de igual manera la asfixia perinatal se encuentra considerada como una condición grave en el neonato, causante de morbilidad y mortalidad, lo cual aumenta el riesgo de presentar algún tipo de discapacidad a largo plazo y con ello, la necesidad de atención especializada, misma que genera costos elevados a nivel económico y social (Torres y Rojas, 2010).

Por otro lado, Narberhaus y Segarra (2004) consideran que actualmente existe una notable escases de datos sobre niños que presentaron complicaciones peri y post natales, dentro de las que se encuentran la prematurez y la asfixia perinatal. Este hecho podría encontrarse estrechamente relacionado con la situación de la prematuridad por sí misma, ya que implica un riesgo más alto de pronóstico no favorable y por tanto, los mecanismos conducentes al mismo pueden ser múltiples debido a la presencia frecuente de complicaciones concurrentes tanto en etapa fetal como en la neonatal.

No obstante, Castro y Barraza (2007) mencionan que al tomar en cuenta el riesgo que implica presentar complicaciones peri y post natales a nivel de desarrollo cognitivo, los estudios a largo plazo con esta población han ido en aumento.

Debido a que los niños con antecedentes de prematuridad representan una población vulnerable a padecer complicaciones al momento del nacimiento, tales como asfixia perinatal, variable de estudio del presente trabajo, resulta de suma importancia investigar el desarrollo en la infancia de los prematuros para así poder llegar a generar intervenciones específicas que tengan utilidad para prevención y rehabilitación de las posibles dificultades funcionales (Ibíd.).

acerca de la situación cognitiva en niños prematuros, resulta de gran importancia explicar que el sistema nervioso central cambia sustantivamente durante el último trimestre de la gestación y el primer mes de vida neonatal, sufriendo modificaciones en su morfología macroscópica cada semana (Castro y Barraza, 2007).

Esta serie de modificaciones consiste en una secuencia de eventos complejos e interrelacionados que posibilitan un adecuado desarrollo cerebral, estos eventos son la migración neuronal, proliferación de células gliares, formación de axones y espinas dendríticas, establecimiento de sinapsis, mielinización, muerte celular programada y estabilización de conexiones corticales (Narberhaus y Segarra 2004).

Para Castro y Barraza (2007) uno de los eventos más importantes entre los antes mencionados es la mielinización, ya que ayuda a pronosticar determinadas manifestaciones neurológicas al ser uno de sus logros fundamentales el conformar una transmisión sináptica precisa y veloz, es por esto que existe una clara relación entre el patrón de mielinización del sistema nervioso y la adquisición de nuevas habilidades funcionales.

El proceso antes mencionado es de suma importancia en los niños prematuros ya que se cuenta con evidencia de alteraciones en su proceso de mielinización, lo que se relacionaría con las importantes dificultades a nivel neurosensorial, motor y cognitivo que presentan, sobre todo en la infancia temprana (Ibíd.).

Debido al desarrollo anormal de los procesos antes mencionados, se logra un sistema nervioso inmaduro incapacitado para otorgar una respuesta adecuada, ajustada y armónica a las diferentes demandas o desafíos del ambiente. Al tener en cuenta lo anterior, pueden aparecer desórdenes en la regulación 


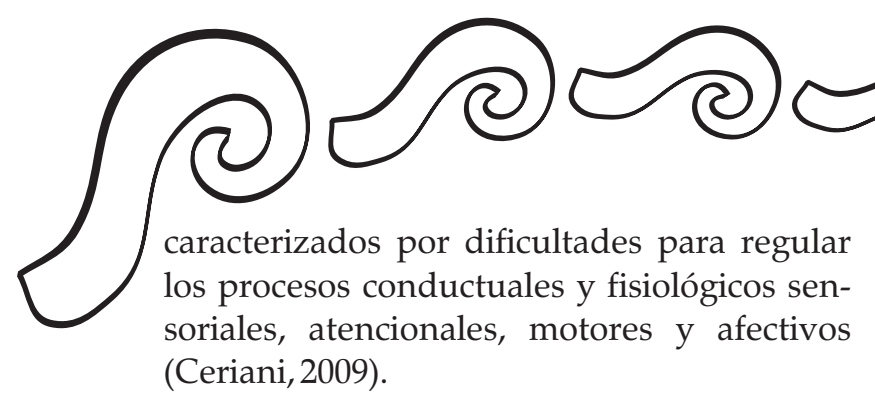

Respecto a esta inmadurez Narberhaus y Segarra (2004) mencionan que es de ella donde derivan los problemas esenciales del prematuro, ya que se hace presente en los diferentes aparatos y sistemas orgánicos, lo que predispone al niño a presentar una serie de complicaciones precoces o tardías.

Como parte de los resultados pertenecientes a los diversos estudios realizados a niños prematuros, se han encontrado evidencias de que esta población presenta de igual manera anomalías funcionales en las primeras semanas de vida postnatal, lo que muestra un descenso del flujo sanguíneo cerebral, así como episodios de descenso de la presión arterial, lo cual situaría al cerebro prematuro en una situación de riesgo para sufrir un accidente hipóxico/isquémico, mismo que ya se ha explicado anteriormente y que en la mayoría de los casos surge como consecuencia de la asfixia perinatal (Ibíd.).

Por otra parte, esos mismos autores refieren que al tomar en cuenta estudios cuantitativos de neuroimagen cerebral, se observa que los niños prematuros presentan anomalías anatómicas cerebrales al nacer, las cuales podrían contribuir a explicar los problemas cognitivos, comportamentales y emocionales que pueden aparecer a largo plazo en los niños prematuros.

Por lo anterior resulta importante considerar la definición de coeficiente intelectual otorgada por Brody (1997, en Cohen, 2001), quien menciona que el coeficiente intelectual (CI) es considerado como un indicador de desempeño en diferentes tipos de tareas intelectuales a nivel cognitivo, es decir, una medida general de una serie de mediciones de las ejecuciones y por medio de lo cual se infieren determinadas habilidades de tipo cognitivo que se traducen en el puntaje de CI.
Existen diversas pruebas que permiten cuantificar las capacidades cognoscitivas y específicas de los individuos; capacidades tales como la abstracción, la capacidad de organización o la habilidad para llevar a cabo tareas mentales diversas. Estas capacidades de la inteligencia son medidas y cuantificadas al utilizar los resultados finales como el índice denominado coeficiente o cociente intelectual (Palencia, 2001).

Al tomar en cuenta estas pruebas, se ha observado que los niños prematuros presentan durante la infancia un déficit en el rendimiento cognitivo general y en ciertas habilidades no verbales, destacando las dificultades en las tareas visoespaciales y visoperceptivas (Narberhaus y Segarra, 2004).

Estos mismos autores hacen referencia de otros estudios realizados con prematuros, mismos en los que se ha encontrado que durante la infancia, es decir, de $\operatorname{los} 3$ a los 8 años, los niños presentan un déficit en el rendimiento cognitivo general, mientras que los déficits neuropsicológicos en la población adolescente son referentes a la lectura y el CI, además del razonamiento matemático.

Por su parte Rendón (2003) menciona que durante la edad escolar los niños con antecedentes de prematuridad presentan pobre rendimiento académico, dificultades del desarrollo y pobre función adaptativa, lo que en conjunto contribuye a un retraso en el neurodesarrollo, asimismo, estos niños presentan dificultades en atención, función ejecutora, memoria, función motora gruesa y motora fina, por lo anterior, esta autora establece una relación entre el daño cerebral perinatal y la funcionalidad posterior.

Debido a la gran importancia de este tema, se han realizado múltiples estudios con la finalidad de ofrecer un panorama más claro sobre esta situación. Uno de estos estudios pertenece a Briscoe y Gathercode (2001, en Narberhaus y Segarra 2004), quienes corroboran la presencia de dificultades no verbales en los 


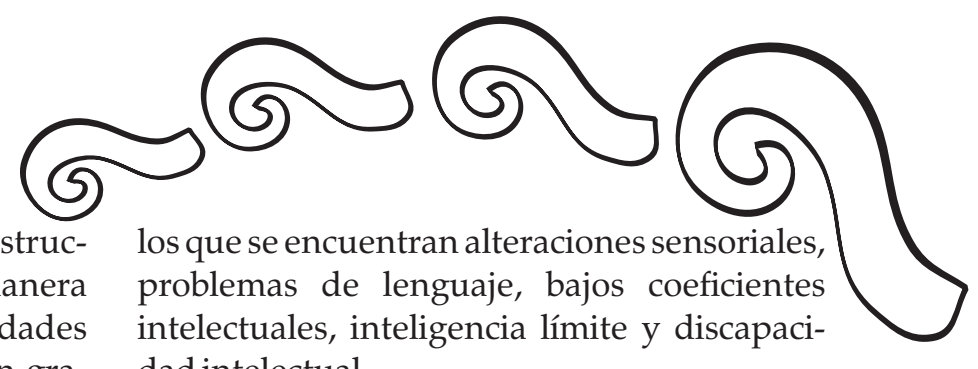

prematuros a través de la tarea de construcción de patrones complejos; de igual manera mencionan la afectación de las habilidades verbales, donde destaca la comprensión gramatical, la comprensión auditiva y la comprensión de órdenes verbales de complejidad creciente.

En contribución a las investigaciones referentes al desarrollo cognitivo, Breslau, Chilcoat, Johnson, Andreski y Lucia (2000, en Castro y Barraza, 2007) realizaron un estudio en el que hallaron que factores tales como el bajo peso al nacimiento y la prematurez representan situaciones que aumentan significativamente el riesgo de obtener un CI medio bajo o limítrofe.

Por otra parte, referente a la asfixia perinatal (s. a.), el estudio longitudinal Avon de Padres y Niños muestra resultados en cuanto a la función cerebral y rendimiento intelectual de 11, 981 niños de ocho años, los cuales tenían antecedentes de prematurez y asfixia perinatal. Esta población se dividió en tres grupos; el primer grupo fue representado por los niños que sufrieron asfixia; el segundo por niños que necesitaron de reanimación y por último, el tercer grupo, conformado por los que respiraron de manera independiente al nacer.

Como parte de los resultados de este estudio, se observó que los niños pertenecientes al primer grupo presentaban un $65 \%$ más de posibilidades de tener un coeficiente intelectual inferior a 80 , es decir medio bajo, mientras que los del segundo grupo se mostraron con mayor vulnerabilidad a desarrollar un nivel intelectual inferior respecto a los neonatos que respiraron sin ayuda.

Por su parte, Garrido y Cordero (1992) documentan que en niños con antecedentes de hipoxia perinatal se han detectado una amplia serie de problemas, tales como mala coordinación, parálisis cerebral, dificultades en motricidad fina y gruesa, convulsiones y afectaciones a nivel de procesos psicológicos, dentro de

\section{dad intelectual.}

\section{Método}

Se realizó un estudio de tipo retrospectivo y transversal al indagar sobre hechos del pasado y no dar seguimiento al fenómeno estudiado.

La población que conforma el presente estudio fue seleccionada mediante muestreo no probabilístico accidental, en el cual se tomaron en cuenta a 18 niños con edades entre los 3 y 12 años que son atendidos por el área de psicología dentro del Centro de Rehabilitación Integral de Hidalgo (CRIH). La población fue elegida al tomar en cuenta la presencia de asfixia perinatal al momento de nacimiento aunado a una edad gestacional prematura.

Como parte de los criterios de elección para la población se tomó en cuenta lo siguiente:

Criterios de inclusión: niños y niñas con edades entre los 3 y 12 años que son atendidos por el consultorio 1 del área de psicología dentro del Centro de Rehabilitación Integral de Hidalgo (CRIH), que presentaron asfixia perinatal al momento de nacimiento aunado a una edad gestacional prematura.

Criterios de exclusión: niños y niñas menores de 3 años o mayores de 12 años que no fueron atendidos dentro del área de psicología del Centro de Rehabilitación Integral de Hidalgo (CRIH), que su edad gestacional fue de 38 semanas o mayor y que no presentaron asfixia perinatal.

Para la realización de la presente investigación sobre el coeficiente intelectual en niños de edad escolar se utilizaron las Escalas de Inteligencia Weschler tanto para los niveles preescolar y primario (WPPSI-III) como para niños (WISC-IV). Cabe mencionar que la últi- 


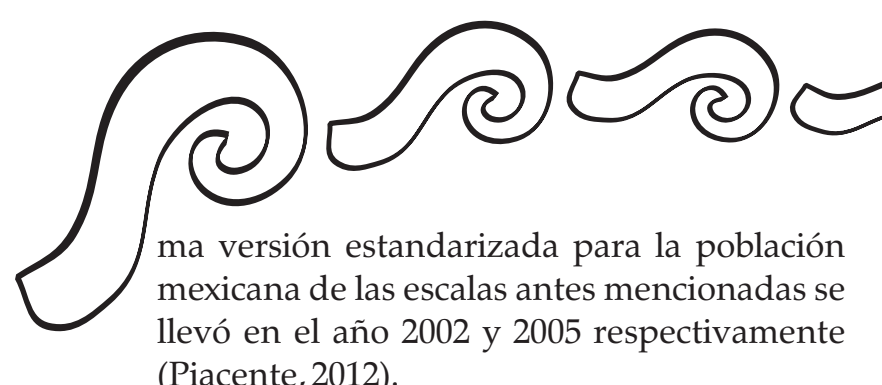

(Piacente, 2012).

La edad comprendida para la Escala de Inteligencia para los niveles preescolar y primario se encuentra entre los 2 años 6 meses y los 7 años 3 meses, sin embargo, al tomar en cuenta el gran cambio en cuanto al desarrollo cognitivo que se presenta en los niños de esta etapa, se decidió dividir la prueba en dos intervalos de edad: el primer intervalo comprende de los 2 años 6 meses a los 3 años 11 meses, mientras que el segundo va de los 4 años a los 7 años 3 meses.

Para el primero de estos intervalos son posibles cuatro puntuaciones compuestas, las cuales son el coeficiente intelectual verbal (CIV), coeficiente intelectual de ejecución (CIE), coeficiente intelectual total (CIT) y el compuesto general del lenguaje (CGL). Mientras que para el segundo las puntuaciones compuestas posibles de obtener son el coeficiente intelectual verbal (CIV), coeficiente intelectual de ejecución (CIE), coeficiente intelectual total (CIT), el compuesto general del lenguaje (CGL) y por último, el cociente de velocidad de procesamiento (CVP).

Por otra parte, para la Escala de Inteligencia para niños (WISC- IV), la edad de aplicación se encuentra entre los 6 años 0 meses y los 16 años 11 meses, proporciona una medida del funcionamiento intelectual general (CIT), además de cuatro puntuaciones índice, las cuales son el Índice de Comprensión Verbal (ICV), Índice de Razonamiento Perceptual (IRP), Índice de Memoria de Trabajo (IMT) y el Índice de Velocidad de Procesamiento (IVP).

Una vez que la recolección de datos referentes a los resultados de las pruebas y de los expedientes fue culminado, se procedió a realizar el análisis cuantitativo y cualitativo para cada uno de los integrantes de la población de la presente investigación.
Como parte del análisis cuantitativo fueron utilizados los constructos estadísticos de medidas de tendencia central y estadígrafos no paramétricos como son: el estadígrafo de Kruskal- Wallis y el estadístico de contraste ${ }^{2}$, mientras que para el análisis cualitativo se hizo uso del análisis de contenido.

Cabe señalar que para el análisis de ambas variables y con la finalidad de obtener datos con mayor exactitud, se utilizó el Statistical Package for the Social Sciences (SPSS) versión 21.0. para llevar a cabo el procesamiento de los datos.

\section{Resultados}

El análisis comprendido en la presente investigación se encuentra dividido en análisis cuantitativo y análisis cualitativo.

En relación al análisis cuantitativo de los datos, resulta importante mencionar que se utilizó tanto la estadística descriptiva como la inferencial. La primera de éstas se utilizó para conocer los resultados más relevantes de la población respecto a cada una de las escalas, mientras que la segunda fue utilizada para la comprobación de las hipótesis planteadas.

Respecto al análisis cualitativo, fueron utilizadas las historias clínicas de cada uno de los sujetos, se hizo uso de la técnica de análisis documental para poder examinar la información de cada sujeto de forma detallada.

El análisis estadístico cuantitativo mostró que de los 18 pacientes que formaron parte del estudio, $28 \%$ fueron niñas y $72 \%$ niños. Por otra parte, al clasificar la edad de los participantes en tres diferentes intervalos, se pudo identificar que la mitad de la población se encuentra en el rango de edad entre 6 y 8 años, continúa con siete sujetos dentro del intervalos de 3 a 5 años y finalmente entre 9 y 12 años únicamente se encuentran 2 .

Al tomar en cuenta la distribución por edad, el $56 \%$ de la población fue valorada mediante la 


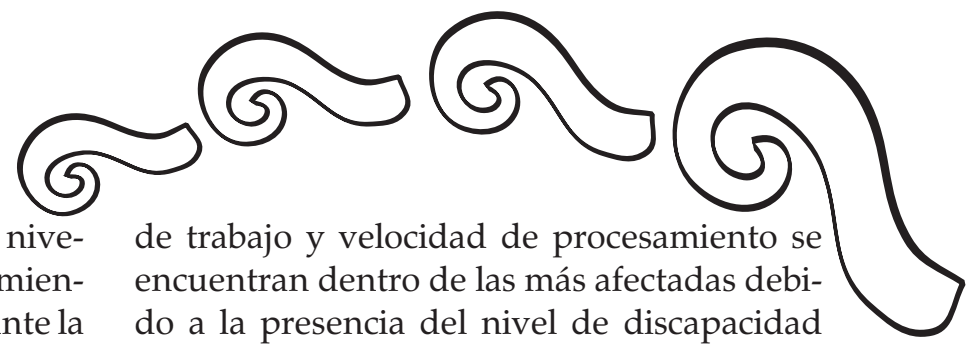

Escala de Inteligencia Weschler para los niveles preescolar y primario (WPPSI- III), mientras que el $44 \%$ restante se valoró mediante la Escala de Inteligencia Wechsler para el Niños (WISC-IV).

Como parte de los resultados arrojados por la aplicación de las Escalas Weschler tanto WPPSI -III como WISC- IV respecto al coeficiente intelectual total de cada uno de los niños participantes dentro de la investigación, fue posible identificar la existencia de mayor incidencia en los niveles: limítrofe y discapacidad intelectual moderada, duplicando la primera al nivel promedio bajo y triplicando a quienes obtuvieron un nivel promedio.

En relación al coeficiente intelectual verbal, éste representa una escala comprendida dentro de la Escala Weschler WPPSI - III. En los resultados arrojados por dicha escala se observa una prevalencia equitativa en el nivel promedio, limítrofe y discapacidad intelectual ligera, lo que triplica a su vez a la cantidad respecto al nivel promedio bajo. Cabe resaltar que ninguno de los sujetos evaluados presentó un nivel promedio alto, superior o muy superior en dicha escala.

Como parte de la Escala Weschler WPPSI-III se encuentra igualmente el coeficiente intelectual de ejecución, mismo en el cual la población se encuentra concentrada mayormente en los niveles promedio bajo, limítrofe y discapacidad intelectual ligera, mostrando mayor incidencia en el nivel promedio bajo y al ser escasos los sujetos ubicados dentro de los niveles promedio y promedio bajo.

Por otra parte, el razonamiento perceptual pertenece a la Escala de Inteligencia Wechsler WISC-IV y en el caso de la población estudiada se identificó mayor prevalencia en los niveles promedio bajo, limítrofe y discapacidad intelectual ligera, mostrándose más acentuada esta última.

Las escalas de comprensión verbal, memoria Respecto a la escala de comprensión verbal se observa que la mayoría de la población se encuentra concentrada en los niveles de discapacidad intelectual, por lo que se muestra repartida en las mismas cantidades en discapacidad intelectual ligera y moderada, donde se observa una disminución en el nivel promedio bajo.

Debido a las características de las afecciones de asfixia prenatal, prematurez y estas dos en conjunto, la cantidad de sujetos evaluados fue limitada, por tal, los análisis inferenciales fueron realizados con estadígrafos no paramétricos. Mediante la aplicación de la prueba de Kruskal - Wallis y su posterior contraste con el estadígrafo de ${ }^{2}$ se encontraron diferencias significativas en la escala de velocidad de procesamiento, al ser ésta el área que más se encuentra afectada en los niños con dichos padecimientos; en especial entre los que coexisten ambos (Tabla 6 y 7).
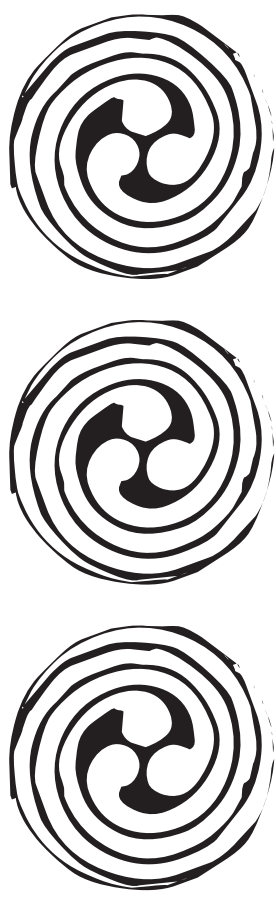


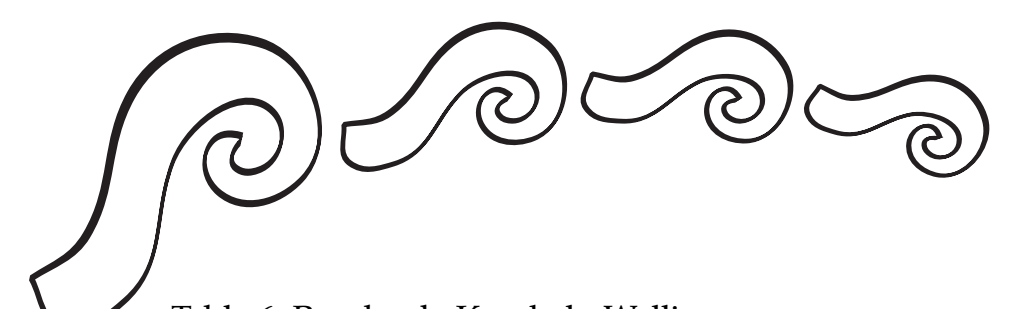

Tabla 6. Prueba de Kruskal - Wallis

\begin{tabular}{|c|c|c|c|}
\hline Escala & Afección & $\mathrm{N}$ & Rango promedio \\
\hline \multirow{4}{*}{$\begin{array}{l}\text { Coeficiente } \\
\text { Intelectual } \\
\text { Total }\end{array}$} & Asfixia & 8 & 9.81 \\
\hline & Prematurez & 4 & 11.25 \\
\hline & Asfixia y prematurez & 6 & 7.95 \\
\hline & Total & 18 & \\
\hline \multirow{4}{*}{$\begin{array}{l}\text { Comprensión } \\
\text { Verbal }\end{array}$} & Asfixia & 2 & 6.50 \\
\hline & Prematurez & 2 & 3.50 \\
\hline & Asfixia y prematurez & 4 & 4.00 \\
\hline & Total & 8 & \\
\hline \multirow{4}{*}{$\begin{array}{c}\text { Razonamiento } \\
\text { Perceptual }\end{array}$} & Asfixia & 2 & 5.50 \\
\hline & Prematurez & 2 & 4.00 \\
\hline & Asfixia y prematurez & 4 & 4.25 \\
\hline & Total & 8 & \\
\hline \multirow{4}{*}{ Memoria de Trabajo } & Asfixia & 2 & 5.50 \\
\hline & Prematurez & 2 & 4.00 \\
\hline & Asfixia y prematurez & 4 & 4.25 \\
\hline & Total & 8 & \\
\hline \multirow{4}{*}{$\begin{array}{l}\text { Velocidad de } \\
\text { procesamiento }\end{array}$} & Asfixia & 7 & 11.14 \\
\hline & Prematurez & 4 & 11.25 \\
\hline & Asfixia y prematurez & 6 & 5.00 \\
\hline & Total & 17 & \\
\hline \multirow{4}{*}{$\begin{array}{l}\text { Coeficiente } \\
\text { Intelectual } \\
\text { Verbal }\end{array}$} & Asfixia & 6 & 4.50 \\
\hline & Prematurez & 2 & 9.50 \\
\hline & Asfixia y prematurez & 2 & 4.50 \\
\hline & Total & 10 & \\
\hline \multirow{4}{*}{$\begin{array}{l}\text { Coeficiente } \\
\text { Intelectual } \\
\text { de Ejecución }\end{array}$} & Asfixia & 6 & 5.00 \\
\hline & Prematurez & 2 & 6.00 \\
\hline & Asfixia y prematurez & 2 & 6.50 \\
\hline & Total & 10 & \\
\hline
\end{tabular}




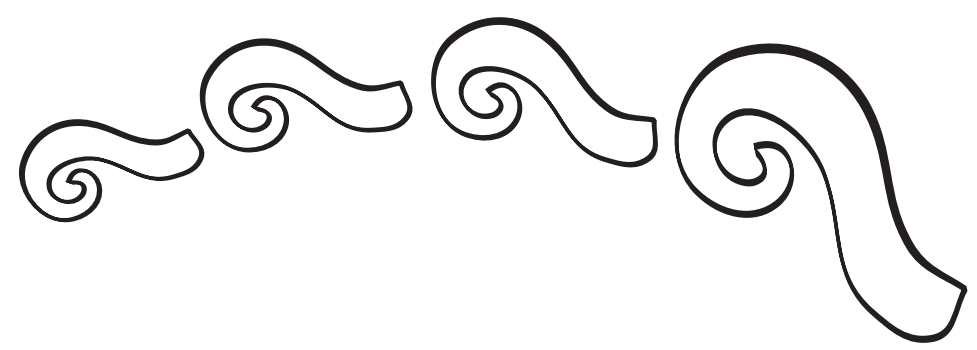

Tabla 7. Estadístico de contraste $\chi^{2}$

\begin{tabular}{|c|c|c|c|c|c|c|c|}
\hline & $\begin{array}{l}\text { Coeficiente } \\
\text { Intelectual } \\
\text { Total }\end{array}$ & $\begin{array}{c}\text { Comprensión } \\
\text { Verbal }\end{array}$ & $\begin{array}{c}\text { Razonamiento } \\
\text { Perceptual }\end{array}$ & $\begin{array}{l}\text { Memoria } \\
\text { de } \\
\text { Trabajo }\end{array}$ & $\begin{array}{c}\text { Velocidad } \\
\text { de } \\
\text { procesamiento }\end{array}$ & $\begin{array}{l}\text { Coeficiente } \\
\text { Intelectual } \\
\text { Verbal }\end{array}$ & $\begin{array}{c}\text { Coeficiente } \\
\text { Intelectual } \\
\text { de } \\
\text { Ejecución }\end{array}$ \\
\hline $\begin{array}{c}\text { Chi } \\
\text { cuadrado }\end{array}$ & .991 & 1.833 & .458 & .464 & 5.848 & 4.364 & .436 \\
\hline $\begin{array}{c}\text { Sig. } \\
\text { asintótica }\end{array}$ & .609 & .400 & .795. & .793 & $.050^{*}$ & .113 & .804 \\
\hline
\end{tabular}

${ }^{*}$ Las diferencias significativas en las escalas de coeficiente intelectual, se encuentran indicadas con *.

El estudio de la parte cualitativa de esta investigación se realizó con el uso de la historia clínica de cada uno de los sujetos (como ya se había referido anteriormente), misma que fue referida por la madre o por algún familiar cercano mediante una entrevista estructurada. El análisis documental fue la técnica usada para el estudio de estos datos. Para poder llevar a cabo el análisis cualitativo se realizará el análisis documental, sujeto por sujeto organizado de la siguiente manera:

Se presenta una primera parte concerniente a la edad y escolaridad del sujeto, posteriormente, en el apartado de antecedentes heredo/familiares se colocan las enfermedades, síndromes o trastornos padecidos por familiares. A continuación, en el apartado referente a antecedentes pre y peri natales se incluye la información acerca del embarazo y alumbramiento. En la categoría de desarrollo de la salud se incluyen algunas enfermedades de importancia padecidas por el sujeto, así como su desarrollo en los primeros años de vida, la presencia de hospitalizaciones y convulsiones.
Siguiente a la categoría antes mencionada, se incluye la dinámica familiar actual del sujeto, el recorrido escolar desde su ingreso al ámbito escolar y su situación actual, y finalmente la integración de su diagnóstico psicológico. Es importante mencionar que con el objeto de mantener el anonimato de los sujetos, serán nombrados con números.

\section{Discusión}

De acuerdo a lo observado en cada una de las diversas escalas, es posible referir que en general cada una de las áreas antes descritas se encuentran afectadas significativamente, por lo que coincide con lo encontrado tanto por Narbehaus y Segarra (2004), Rendón (2003), así como Garrido y Cordero (1992), respecto a la existencia de un déficit cognitivo general tanto en niños prematuros como en aquellos con antecedentes de asfixia perinatal.

Por lo anterior, una vez realizado el análisis de los resultados y su respectiva vinculación con la teoría, se comprueba la existencia de una afectación en el coeficiente intelectual total, así como en velocidad de procesamiento, 


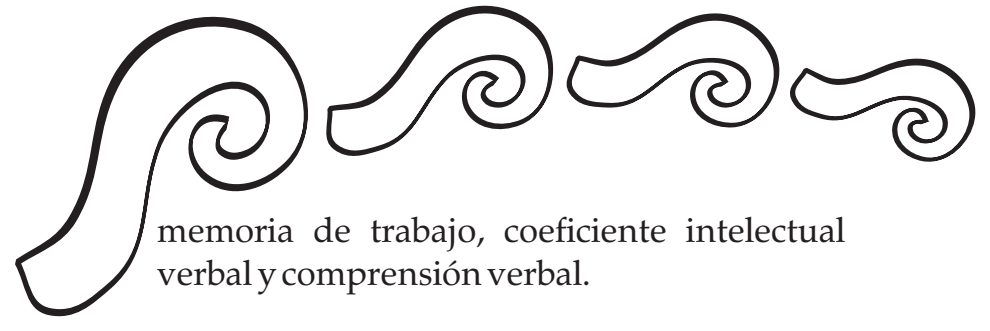

De manera particular, se considera que los resultados cualitativos aportados por el análisis documental enriquecen en gran medida a los datos cuantitativos, ya que al tomar en cuenta datos cualitativos se obtiene una visión más amplia de cada uno de los sujetos partícipes de la investigación, lo que otorga información que las pruebas psicométricas no brindan y que su conocimiento es de vital importancia.

Ya que el análisis se realizó de manera más completa, es posible establecer que las áreas específicas de afectación en los niños con antecedentes de prematurez y asfixia perinatal basadas en el análisis cualitativo son:

- Alteraciones en cuanto a comprensión verbal y atención

- Madurez mental inferior

- Retardo en el desarrollo psicomotor grueso

Aunado a lo anterior, igualmente se identificaron algunas características comunes presentes en los casos:

- Familiares con problemas de aprendizaje

- Familiares con problemas de lenguaje

- Control prenatal tardío

- Demanda de afecto

- Inseguridad

Por lo anterior resulta de gran importancia el realizar investigaciones con un incremento en el tamaño de la muestra haciendo partícipe a población de diferentes instituciones con la finalidad de corroborar los resultados expuestos en el presente trabajo, encontrar diferencias y con lo anterior otorgar mayor contundencia a los resultados sin dejar de lado la utilización del análisis documental, ya que esto le permitirá al investigador explorar diversas características de importancia dentro del análisis global de los resultados.
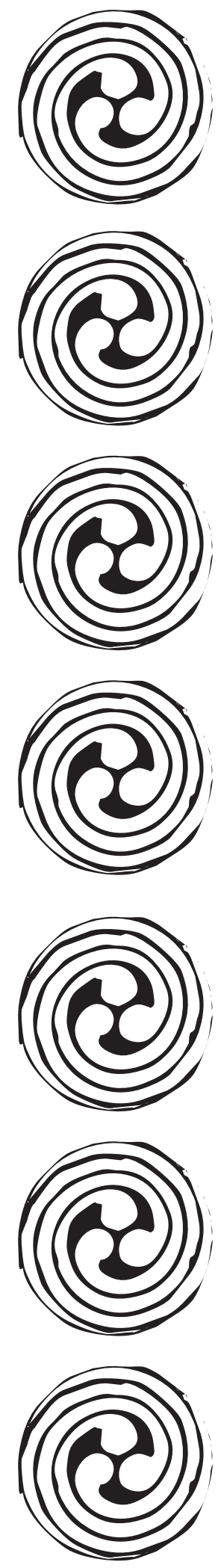


\section{Fuentes de consulta}

Castro, P. y Barraza, P. (2007). Diferencias cerebrales en prematuros y su relación con el desarrollo de sus funciones cognitivas. Terapia psicológica, 25(002), 183-187.

Ceriani, J. M. (2009). Neonatología práctica. Buenos Aires: Medica Panamericana.

Cohen, L. (2001). Influencia de las interacciones familiares y escolares en el uso de conectivos en niños escolarizados. (Tesis inédita de maestría). Universidad de Sonora, Sonora, México.

Delfino, A., Weinberg, M., Delucchi, G., Del Campo, S., Bargueño, M., Filgueira, L., Giró, N., Grajales, M.I., Juncal, A., Kessler, P., Lanwagen, M., Mancuello, K., Marichal, V., Vargas, L., Yanes, M., Bengoa, S., Berta, S. y Scavone, C. (2010). Seguimiento de recién nacidos con asfixia perinatal. Archivo Pediátrico Uruguayo, 81(2), 73-77.

Narberhaus, A. y Segarra, D. (2004). Trastornos neuropsicológicos y del neurodesarrollo del prematuro. Anales de Psicología, 20(2), 317-326.

Palencia, L. F. (2001). Las pruebas psicométricas para la determinación del coeficiente intelectual en el campo de la psicopedagogía. (Tésis inédita de licenciatura). Universidad Nacional Autónoma de México, México D.F.

Rendón, M. C. (2003). Prevalencia del déficit neurológico en el paciente con antecedentes de prematurez egresado de la unidad de cuidados intensivos neonatales del hospital central norte de petróleos mexicanos. (Tesis inédita de posgrado). Universidad Autónoma de México, México.

Torres, J. y Rojas, C. (2010). Asfixia perinatal. Programa de Educación Continua en Pedriatría, 9(3), 17 $-27$.
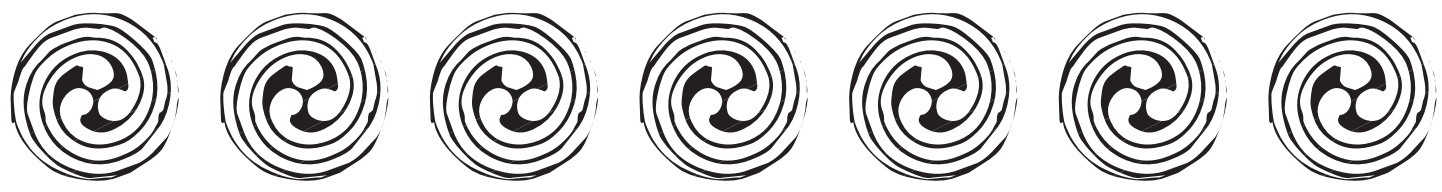

๑ $82 @$ 